\title{
SEVENTH DISCUSSION SESSION
}

(Saturday afternoon; 9 September, 1972)

\section{Chairman: D. H. MCNAMARA}

McNamara: We'll open our session this afternoon with a few remarks by Dr. Cooper on infra-red observations of binary stars.

Cooper: I want to draw attention to the value of infra-red observations of binary systems in improving our understanding of these objects. Semi-detached systems which contain an early-type, main-sequence component and a cooler subgiant will display more nearly comparable depths of their two minima at longer wavelengths: therefore, solutions of their light curves will be more determinate. Other areas in which infra-red observations will be useful are:

(i) determination of limb-darkening coefficients and comparison of the results with those of model-atmosphere calculations (Grygar, 1972).

(ii) study of the atmospheres of components of systems that have been observed in many colours - in particular the atmospheres of cooler components of Algol-type binaries.

(iii) investigation of the possible variation of deduced values of some orbital elements (e.g. radius of eclipsed component) with the effective wavelength in which observations were made.

(iv) improvement of the determination of orbital elements so that one can also obtain a better idea of streaming effects.

(v) investigation of systems of the type of $\varepsilon$ Aur, and of the composition and structure of the disks found in these systems; possibly detection of a secondary minimum.

Smak: I want to emphasize one point, in particular, made by Dr. Cooper: namely that one should try to observe some systems in which the complications arising from circumstellar matter are not severe, in order to study the physical properties of the secondary component.

Andersen: How faint a star will you be able to observe, and what wavelength will you use?

Cooper: I will probably observe at $0.9 \mu \mathrm{m}$ or $2.0 \mu \mathrm{m}$.

McNamara: How faint can you reach with, say, a 24-inch or 50-inch telescope?

Milone: Several of us have made intermediate-band infra-red observations, including the $K$ band, with 50-inch and 60-inch telescopes in Arizona. My recollection is that we could work on objects of at least ninth visual magnitude, and still have reasonable signal-to-noise ratios.

Chen: Before we go on to discuss ultra-violet excesses in binary stars, I would like to point out that although the Harvard and Arizona astronomers have done much work on the infra-red excesses of single stars, we know very little about infra-red excesses of close binary stars. Thus I would like to emphasize once more the need for infra-red observations. 
McNamara: If there are no more questions on this topic, we will ask Dr. Hall to discuss ultra-violet excesses in close binary stars.

Hall: It is somewhat surprising that we still do not know for sure how to interpret the apparent ultra-violet excesses found in the subgiants of totally eclipsing Algol-type binaries. In our paper on SW Cyg (Hall and Garrison, 1972), we spent some time explaining why it is most reasonable to attribute at least part of the excess to contamination by light from an envelope surrounding the hot star, that is not completely eclipsed at mid-primary minimum. Briefly, the evidence in favour of this interpretation is as follows: (i) the amount of the excess is often variable; (ii) in the light curve of SW Cyg the bottom of primary eclipse appears total in $V$ but is so rounded in $U$ that it appears partial; (iii) some of the excesses are larger than 0.2 or 0.3 and hence larger than the maximum excess which would result in the limit of vanishingly small metal abundance. It is still quite possible, however, that the excesses 0.2 or smaller can be the result of a true underabundance of metals, as seems to be indicated by the narrow-band photometry of Miner (1966) and McNamara (1967). Even though this narrow-band photometry was done almost ten years ago, we still cannot say for sure whether or not there is a true metal underabundance in the subgiants. The answer is of interest because there are a number of reasons to expect normal abundance or overabundance of metals, but no good reason to expect an underabundance.

The right way to settle the question would be to obtain a fairly good spectrogram during totality of some Algol-type eclipsing binary with an apparent ultra-violet excess of about 0.2 . Then an abundance analysis, or perhaps even visual inspection, should reveal whether or not the subgiant is truly underabundant in metals. Dr. McNamara and I together want to ask participants if anyone has such spectra and can settle the question.

Baldwin: I investigated this for the secondary component of $U$ Cep, using a spectrogram of $40 \AA \mathrm{mm}^{-1}$ obtained during totality by Dr. Batten. This was the highest dispersion at which we could obtain a good spectrogram. I didn't find the difference that Miner found. I made only a qualitative comparison, with several spectrograms at the same dispersion of single stars of the same spectral type and luminosity class. Visual comparison of the spectrograms showed no significant differences between the stars, so it seems unlikely that there are significant abundance differences.

Popper: There is one observation of another system, not exactly of the same type, namely AR Lac. During total eclipse, Spinrad obtained scans in his multi-channel system and he finds a definite underabundance of metals in the subgiant secondary of AR Lac.

Hall: In my interpretation those are different types of stars, although there may be some uncertainty about that. Dr. McNamara, did you say that an underabundance as large as that implied by narrow-band photometry should 'hit you in the face' spectroscopically?

McNamara: Yes, if you have ultra-violet excesses of the order of 0.2 . Usually in the extremely metal-poor stars, of course, the ultra-violet excesses are of the order of 
$0^{\mathrm{m}}$.2. So in the eclipsing stars, regardless of whether this ultra-violet excess is caused by the filling in of lines by emission or radiation from some source, I should think that the behaviour of very strong lines would mimic what you see in the spectra of metalpoor stars. The deep absorption lines ought to be filled in, and you should be able to notice this in the spectrum of the secondary star. Now for U Cep, $\Delta m_{1} \sim 0$ m 10 . If this implies that the star is metal-poor, I'm almost positive that you ought to observe weak metal lines even at rather moderate dispersion.

D. B. Wood: I made some Strömgren four-colour and magnesium-index observations of some eclipsing stars. One, a W UMa system, had an $m_{1}$ index which, if interpreted as an abundance indicator, showed that the metal abundance varied, from one season to the next, by an order of magnitude. My interpretation is that the $m_{1}$ index is not an indicator of metal abundance in this case.

Baldwin: There was one difference between the secondary component of U Cep and my comparison stars. The $\mathrm{H}$ and $\mathrm{K}$ lines of calcium appeared to be filled in by emission. None of the other lines, at least on visual inspection, showed any significant difference.

McNamara: If I remember correctly, Miner's observations were based on the G-band and the CN-band, and a metal-abundance index $m^{\prime}$. He found all the late-type subgiant components were metal-poor compared with other stars of similar temperature and luminosity class.

Andersen: You said you compared the spectrum with spectra of similar type. Could a low metal abundance disguise itself as a wrong spectral type?

Baldwin: That is something one must be careful about, but the close correspondence of all the stellar lines seemed to indicate that the assigned spectral type and luminosity class were correct.

Batten: We have the photometric solution for U Cep, and, as we now believe, a reasonable idea of the total mass of the system. This gives us the radius of the secondary component, and it is definitely a subgiant, or even a giant.

McNamara: If these stars really are underabundant in heavy elements, it does do damage to the idea of unloading mass from the subgiant components on to the brighter B-type or A-type stars in these systems. If you examine the spectra of the A-type or B-type components, you find, of course, that they have no apparent anomalies; whereas, the indices, on the other hand, if you believe that they really do measure abundances, indicate that there are some rather severe abundance anomalies in the subgiant components. If there are no more questions on this topic, I think we can turn to the contribution by R. E. Wilson and Devinney on common envelopes in close binary stars.

R. E. Wilson: Somewhat more than a year ago we published a procedure for computing light curves of synchronously rotating binaries. The scheme is quite general in that it can be used for detached, semi-detached, or fully contact systems. The model we use is very similar to that used by certain other groups and individuals (i.e. Lucy (1968), Hutchings and Hill (1973), Mochnacki and Doughty (1972) Ruciński (1969)) but the computing scheme is more accurate than the others mentioned, by at 
least an order of magnitude. For differential correction of such light curves, it is quite crucial to have the greatest achievable accuracy because some of the numerical derivatives (those for parameters of a geometrical nature) can be computed accurately only when the underlying basic function is extremely smooth. The computational errors in our light curves are small compared to the accidental errors of the best normal-point observations. The present work (and its continuation) has three objectives, which are:

(1) To establish whether the W UMa binaries are true contact systems, having common envelopes.

(2) To establish the gravity darkening law which holds for stars which have convective envelopes.

(3) To begin collecting fundamental data for contact systems (i.e. masses, luminosities, and radii).

The first two points are, of course, observational checks on ideas advanced by Lucy. Work on the third point has been hampered until recently by the shortcomings possessed by the classical Russell model when it is applied to close binaries. Not only does the Russell model fit poorly to observed W UMa light curves but the derived values of the parameters are systematically in error by relatively large amounts (e.g. $5^{\circ}-7^{\circ}$ in the inclination, $\sim 20 \%$ in the radii). This is particularly unfortunate since many W UMa systems are double-lined spectroscopic binaries with large radial velocity amplitudes, so that the prospect for obtaining absolute dimensions is good from the spectroscopic point of view. Furthermore, the spectroscopic information can be supplemented by accurate photometric mass ratios derived from the new approach.

The adjustments presented here were produced by application of our differentialcorrections program, following preliminary trial-and-error fitting. The differentialcorrections program makes a least-squares solution for observations in an arbitrary number of passbands simultaneously. The solution is rigorous in the sense that only one value is found for each wavelength-independent parameter while for wavelengthdependent parameters, $n$ values are found for the $n$ passbands. Of course, the program computes probable errors for all adjusted parameters.

For contact binaries, constraints are imposed on the parameter values such that there are no discontinuities in surface brightness across the neck connecting the two components. The set of adjustable parameters for contact systems includes:

$q=$ mass ratio $\left(m_{2} / m_{1}\right)$,

$i=$ orbital inclination,

$g$ = gravity darkening exponent,

$\boldsymbol{\Omega}=$ modified potential for photosphere,

$x=$ limb darkening coefficient,

$A=$ bolometric albedo,

$l_{3}=$ third light,

$T_{1}=$ polar temperature of component 1 .

Only the first five parameters were adjusted for the present examples. In addition to the above list, the luminosity ratio, $L_{1} / L_{2}$, and polar temperature, $T_{2}$, of component 2 
are computed, but these are not free parameters since they follow from the values assigned to the other quantities. Binaries for analysis were selected for having total or annular eclipses, absence of asymmetries, and accurate observations. We have solved for the gravity exponent by least squares rather than by assuming that the 'convective' value predicted by Lucy is correct, as did Mochnacki and Doughty. Short comments on our results for three systems follow:

$R Z$ Com - Green light curves taken at two different epochs by Broglia (1960) were analyzed independently. This system seems to be only marginally contact, as the solution showed it to be slightly over contact at one epoch and slightly under at the other. The two light curves are significantly different by visual inspection. The values found for $g$ were $1.13 \pm 0.04$ and $1.51 \pm 0.02$ for the two light curves ( $g$ is 1.00 for Von Zeipel darkening, $\sim 0.32$ for Lucy darkening).

$R Z$ Tau - Contact is definitely indicated for this binary. The flat annular eclipse shown by Binnendijk's (1963) light curve could not be reproduced without destroying the fit in other phase ranges. Conceivably this apparent flat region could be due to one or two unfortunate residuals, and may not be found in future light curves. The gravity darkening, $g$, was $0.29 \pm 0.06$. The mass ratio we find is close to that found by Mochnacki and Doughty, who also studied this binary, but several other parameters are considerably different, especially the limb-darkening coefficients.

$A W U M a$ - The components are certainly in contact for this system. $g$ is $0.45 \pm .06$ and the mass ratio is close to that found by Mochnacki and Doughty, who did not adjust the limb-darkening for this system.

Chen: I would like to mention that models for W UMa-type stars were discussed in great detail by Lucy and Mauder at the Philadelphia meeting last year. In fact, all the items listed by Wilson and Devinney were fully discussed there. Lucy has given solutions for W UMa-type light curves. It would be interesting to compare his results with yours. Lucy pointed out, as noted by others, that the eclipse of a larger mass always gives a deeper eclipse. This is related, of course, to the problem of discrepancies between the spectroscopic mass ratio and the photometric mass ratio for some systems.

R. E. Wilson: Well, I was not at the Philadelphia meeting, and the Proceedings haven't been published yet. Perhaps you can tell me if Lucy's results were obtained by differential corrections. I presume they were based on the program that he has already published (Lucy, 1968).

Hill: Lucy wrote a special program, in differential-correcting form, for the Philadelphia conference.

R. E. Wilson: And what was the light-curve program? Basically the one that he had before?

Hill: I think so. I think it was similar to the one published recently by Mochnacki and Doughty.

R. E. Wilson: Well, that program was quite different from ours. The advantage of our program is that it has considerably higher precision than have other existing programs for light curves of close binaries, and therefore the errors are completely negligible compared to the errors of even the best normal points. This is important in 
a differential-corrections program, because when you're computing numerical derivatives, the basic function must be extremely smooth if you are to obtain reliable results.

Biermann: I would like to mention that this is the fourth determination of an extremely high mass ratio for AW UMa. Lucy did write a special program for the Philadelphia meeting, and for this system he found a mass ratio of about 12:1. Mauder (1972) published recently a long list of such determinations, and I believe he got a similar result. There are a number of recent papers by the Sussex group [Hazlehurst (1970), Moss and Whelan (1970) and by Dr. Thomas and myself (unpublished)] which discuss several possibilities for what really happens in the common convective envelope. In our paper, Thomas and I indicate what we should like to have observed - especially binaries in clusters, a problem which apparently John Whelan is tackling. Theoreticians, both in Europe and the United States, have provided a variety of models which occasionally disagree violently in their predictions. Observers like you could help to decide among them.

Smak: I wish to add one more comment that is extremely important for the theory, namely, you should note the degree of contact and try to interpret your result. You can get a certain degree of overflow of the inner contact surface. You don't get too close to the outer contact surface. That may indicate that in those cases any major mass outflow from the system is unlikely.

R. E. Wilson: For AW UMa, the photosphere is about half-way between the inner and outer contact surfaces.

Herczeg: I should like to put a mild objection on the record. Someone said that W UMa stars are well-behaved objects, offering no serious observational difficulties. This is certainly an over-optimistic opinion. Everybody who has studied their spectra knows that the lines are rather badly broadened and blended. For instance, in many cases we see only a single spectrum which, however, may be blended with the lines of the secondary; thus we observe a reduced amplitude of the radial velocity curve and an incorrect mass function - AK Her is perhaps an example. Also, some of the light curves have a tendency to show irregular, cycle-to-cycle changes. How does Dr. Wilson cope, for instance, with such disturbances? Using two somewhat different light curves, did you get two different sets of elements? If so, do they represent real changes of the system?

R. E. Wilson: Well, the main way in which the sets of elements differed was that the gravity-darkening parameter had a value from one of the curves which, at first sight, seemed physically unreasonable. Since that parameter is related to the brightness distribution over the stars, it seems likely that whatever happened to the system could most conveniently be described in terms of the brightness distribution.

Herczeg: Would you say that in other cases of disturbed light curves this type of variation can also be ascribed to changes in brightness distribution?

R. E. Wilson: Yes, but it's difficult to extrapolate from one example.

Herczeg: Concerning the overflow of the Roche lobe, whether or not it is possible theoretically, one should bear in mind the strange fact that there seems to be a sur- 
prisingly small amount of circumstellar matter in W UMa systems. The radialvelocity curves are symmetrical sine-like curves, as is to be expected for nearly circular orbits. There is no Barr effect observed in W UMa spectra. One observational difficulty is that you need a large telescope, in order to obtain good time resolution. Otherwise you must have an additional program to compute the line deformations due to the radial-velocity change during exposure.

$R$. E. Wilson: That part of the difficulty that arises from rotational broadening can be taken care of by computing the profiles from this type of model. Despite these difficulties, we have a fairly good idea of the mass functions, at least. If on top of this you can obtain a photometric determination of the mass ratios, you ought to be able to determine approximately the right masses.

Biermann: If you do the analysis in more detail, as Thomas and I did, you find that the constant in the gravity-darkening law is not really a constant but varies slightly (from about 0.06 to 0.10 ) for different models of stars. It might be of interest to check your different systems against our values to see whether there is any accord between these determinations. On the other hand, from the theoretical point of view, even detached, near-contact binaries must obey Lucy's type of gravity-darkening law. The law is not a property of contact binaries, but of convective zones.

Smak: I have a somewhat pessimistic remark. In spite of all these beautiful results, we should not forget that the basic models involved fail to explain several important features. One is the asymmetry of the light curve, and the other is the character of primary eclipse which must always be a transit in Lucy's model, but does not always seem to be so in practice.

R. E. Wilson: First of all, the colour effect in AW UMa is very small - probably smaller than you realize. The eclipses are very shallow and the light curve I showed was drawn on a very expanded scale. The differential effect from yellow to blue is less than 0.01 . As to primary eclipse being a transit or occultation; that is associated in our program with the value of the gravity-darkening exponent. A system with classical gravity darkening has primary eclipse as an occultation: with convective gravity darkening that eclipse is a transit. From experiments with light curves, we see that this last statement is generally true. If future experience confirms this, we could tell rather quickly which gravity-darkening law to apply simply by whether primary eclipse is a transit or an occultation.

Whelan: Is the secondary hotter?

$R$. E. Wilson: In all systems the more massive star has the higher polar temperature. Whelan: Really?

R. E. Wilson: It has to.

Whelan: I don't think that's necessarily right. It may be inherent in your gravitydarkening procedure, however.

R. E. Wilson: You may be taking a different approach and not be really considering the same kind of common envelope as I am. If you consider a common envelope in which there are no physical discontinuities of any kind, so that all the way from the pole of one star to that of the other all physical parameters vary smoothly, then the 
temperature has to be higher at the pole of the more massive star simply because gravity is higher there.

Whelan: But if you define primary minimum by the radial-velocity curve, you find that in some systems it is an occultation, and in others a transit. I don't think that you can say that, therefore, in some systems there is radiative gravity-darkening and in others convective. I think you should say: in some systems the primary is hotter, in others the secondary. I am suggesting that theory may predict something that doesn't seem to come out in your program because you represent temperature structure by gravity-darkening rather than by a physical temperature difference between the components with the secondary being the hotter.

R. E. Wilson: You would have to propose then that the physical variables are not consistent with a smooth gravity-darkening law that runs all the way from the pole of one component to that of the other.

Whelan: It's possible that there are discontinuities in the neck between the two stars. The structure of the neck is uncertain to say the least.

R. E. Wilson: You may very well be correct on physical grounds, but the structure of the neck is a problem in stellar interiors. In the first reconaissance of the problem we would like to have a smooth variation of all parameters.

Whelan: Yes, I'm not criticizing your approach to the problem. I'm extremely pleased to see people using light-curve synthesis for these systems. It has already been said here that rectification was not designed to deal with very close binary systems. The question is whether we can still get good parameters. I think the spectroscopy is the clue, but it's not easy to derive values for $K_{1}$ and $K_{2}$. The spectra are very hard to measure.

$R$. E. Wilson: In many systems, especially those with extreme values of the mass ratio, it's far better to take the photometric mass ratio than the spectroscopic. Nevertheless I would be pleased to have the mass function spectroscopically. That's all you need if you have a photometric mass ratio.

Whelan: That's the best way for mass ratios close to unity or to zero. I think many systems have mass ratios between 0.4 and 0.6 . We don't really know because in the past analyses have been based on only a few points on the radial-velocity curve. If mass ratios do lie in that range, and the systems often show shallow eclipses because the inclinations are not very large, then I think that the spectroscopic constraints are the only thing that will enable you to obtain a reasonable solution, because there might be more than one equally good solution in the solution-space domain. The system of TX Cnc is a good example of a case for which you must have good spectroscopy before you can even undertake any sensible photometric solution. If you do have a spectroscopic mass ratio, you can get a good value for the orbital inclination and for the constant, $C$, (that defines the potential surface) and then you can determine parameters for these theoretical models.

R. E. Wilson: All of the systems we have studied have complete eclipses: the problem of partial eclipses does not arise.

Whelan: But some systems do have partial eclipses, and I hope that your program, 
and those of others, will be able to deal with them, so that we can get some very good data! The theory does seem to be in need of observational correction.

Hilditch: What are the formal probably errors of the gravity-darkening exponents you determined for these systems?

R. E. Wilson: For RZ Com we found $1.13 \pm 0.04$ at one epoch, at another we found $1.51 \pm 0.02$. For RZ Tau and AW UMa we found $0.29 \pm 0.06$ and $0.45 \pm 0.06$, respectively. These errors are probable errors, not standard deviations.

Whelan: What would happen if you fixed the exponent $\beta$ at 0.08 , and didn't allow it to reach 1.13? There are good physical arguments for this value of $\beta$. If you fix $\beta$, and allow discontinuities in the neck, and let the secondary temperature be defined by the minima of the light curve, would you find the temperature difference I have suggested? It's a serious problem: if the primary is in front at the deeper minimum, then it may be the cooler component in some average sense. It's important to know if the primary really is the cooler star: it's the more massive and more luminous one. If it is the cooler star, this fact must be tied in with the energy-transfer equation and the theory of these systems.

R. E. Wilson: Without a fundamental change of approach, fixing the gravitydarkening exponent would not lead to a temperature difference. The exponent controls only the amplitude of the effect, and not the sense. The sense of the effect is determined by the fact that the acceleration due to gravity is greater on the more massive component than on the secondary component.

Whelan: O.K. In Lucy's original paper (1968) in which he was trying to use his model to obtain the correct light curves, he demanded equal average effective temperatures for both components, and he found that the minima were the wrong way round. Recently Hutchings and Hill (1973) and Mochnacki and Doughty (1972) have allowed the two stars to have different temperatures. Binnendijk (1965) distinguished two classes of systems - W systems, in which the secondary is hotter, and A systems in which the primary is hotter. This is the same distinction that I made earlier by the radial-velocity curve, which shows which star is receding. Lucy's model does explain the mass-luminosity law in these systems ( $L \propto M$ instead of $\left.L \propto M^{5}\right)$ but it does have this problem. If we can get good observations and good accuracy in our computations we have a very good chance of obtaining some physical insight into theoretical stellar structure.

McNamara: Are there any more questions?

Smak: With so many parameters involved, are you really sure that you obtained them all separately - or may some of them be combined?

R. E. Wilson: First, it is not necessary to use all the parameters if one doesn't want to. You can choose any subset that you would like to adjust. If you feel you have independent information that fixes certain parameters, you can specify a control integer that suppresses their adjustment. Second, we determine the probable errors, taking into account the correlation between parameters. In principle, at least, provided there are no systematic errors, these uncertainties will tell you what you want to know. Finally, we have built into our program the capability to adjust any subset of the par- 
ameters, and in each individual case we adjust only the minimum absolutely necessary.

Van 't Veer: Gravity darkening gives a relation between local temperature and local gravity. At the first Lagrangian point, the gravity is zero, but we know that the temperature is not zero. How do you solve this problem?

$R$. E. Wilson: Where the gravity is zero, the effective temperature is zero and the flux is zero. The Lagrangian point is only a point, so you get negligible flux from a differential volume.

Van 't Veer: Is this physically realistic?

R. E. Wilson: Why not? The problem only arises in a, literally, point-contact binary. Most of the systems we consider are not like that. If we ever do treat a pointcontact binary, then the formal value of the effective temperature at that point is zero, but that does not mean that the local kinetic temperature is zero. We must distinguish between effective and kinetic temperatures. The former varies with local gravity, while the latter is constant (or approximately so in the convective case) over an equipotential surface.

Hutchings: Can I just make a brief comment about the spectroscopic mass ratios? These are based on line profiles which are intrinsically distorted at every phase because of the distortion of the star and because of the variation of temperature across the surface of the star. For the models which we have done for similar types of systems we have calculated this amount of distortion and we are able to reconcile the difference between the spectroscopic and photometric mass ratios. This difference is something one should be aware of and watch out for.

$R$. E. Wilson: And you believe that the photometric values are correct?

Hutchings: I think they are better.

McNamara: One more short remark from Dr. Whelan.

Whelan: With this sophisticated method of solving light curves, people can no longer argue that we can permit errors in our photometry because the theory is not as accurate. You need accurate photometry and good spectroscopy. If you can find systems in clusters, for which we have constraints on chemical composition and distance, then we have a good comparison for theory. So far there's only one in a cluster - TX Cnc - and that's really knocked the theory down. If we could find some more, then I think that this whole business could be placed on a firmer foundation. We have made tremendous advances in understanding the spectroscopy and in the technique of photometric solution.

R. E. Wilson: To go back to your earlier remarks: people computing models for interiors should say what the brightness distribution of the stars should be, and, if there are discontinuities at the neck, what they will be like. Otherwise we cannot know how we should modify the simple brightness distribution used in this model.

Whelan: I agree with that point.

Cooper: Dr. Wilson, when you fit all these parameters, including gravity-darkening to the light curve, was the only criterion to obtain the best possible fit - or did you, for example, choose a less well-fitting solution if, say, the gravity-darkening had a more plausible value? 
R. E. Wilson: The main trouble with contact binaries is to find anything that will fit. After a few tries, the theoretical curves become fairly close to the observations, but to obtain the kind of agreement we obtained requires an enormous amount of trouble. We do not have much flexibility among these parameters. In fact there are fewer parameters than in the conventional Russell model. That has about thirteen parameters, and even when we adjust everything, including third light, we have only seven.

Biermann: In our interior calculations, we compute just the maxima and minima of the light curve. We take each component separately and say: we have this luminosity for that volume. So we have the discontinuity in our program, and this makes it possible for the secondaries to be hotter, as John Whelan has already said. It would be very important for we theoreticians if you could put another step into your program, just to see what luminosities you do get if you allow for the discontinuity. Then you would probably find all these temperature effects.

R. E. Wilson: Well, that could be done very easily, but it introduces another parameter. Recall the problem that Dr. Smak mentioned - that the yellow and blue light curves have slightly different levels at secondary eclipse. That problem will disappear when the extra parameter is added. Then you must judge whether too much weight has been lost from the determination. Of course, if there's a physical reason for believing that that parameter should be accounted for, then it should be added.

Whelan: In principle, scattering of the light at the neck might be detectable by polarization observations. Some of us tried this with Gehrels' excellent equipment at the Lunar and Planetary Laboratory. We could not find any systematic changes in polarization with phase - so we're still stuck because observations do not give the results predicted by theoreticians.

McNamara: Dr. Underhill, is a Cen a binary?

Underhill: Binary stars show a complex spectrum that arises from four sources: (i) spectrum of the primary star, (ii) spectrum of the secondary star, (iii) absorption lines formed during atmospheric eclipse, and (iv) lines (absorption or emission) formed in a gas stream. The problem in devising a model is to recognize which spectral details correspond to which source. One is assisted by considering the relationship of spectral changes to light changes. Klinglesmith and I would propose that $a$ Cen is an eclipsing star and that the occurrence of grazing eclipses is an explanation of what is seen. The light changes are as follows: Norris (1971) has shown that $a$ Cen in visible light is slightly fainter than average around phase 0.0 (He I lines strong) and brighter at phase 0.5 (He I lines weak). Molnar (1972) from observations with OAO-2 has shown that $a$ Cen is faint in the far UV at phase 0.0 and bright around phase 0.5 . The spectroscopic data [Norris (1971), Klinglesmith et al. (1971) and my own observations] show the following: (i) the Balmer lines remain practically constant in shape and strength throughout the cycle and the Balmer series breaks off at $n_{m}=17$. There is no evidence of density changes; (ii) the $\mathrm{He} \mathrm{I}$ lines vary in intensity in a period of 8.814 from stronger than at type B2 to as weak as in type B8 or B9. The triplets maintain a sharp core; the singlets do not; (iii) a set of sharp lines (C II, O II, Si II, Si III, Mg II, Fe III) is present which varies slightly in position and strength. These 
lines seem to strengthen around phase 0.5 , ( $\mathrm{He}_{\mathrm{I}}$ minimum); (iv) broad shallow lines of $\mathrm{Fe} \mathrm{I}$ appear near phase $0^{P} .0$ and remain strong until at least phase 0.25 . There are no broad shallow lines of $\mathrm{Ca}$ II or Fe II at any time.

We suggest that the Balmer lines, the weak $\mathrm{He} \mathrm{I}$ lines and the sharp lines are produced by the primary star which is a large expanded object. The strong He I lines and the $\mathrm{Fe} \mathrm{I}$ lines come from the secondary star which is a compact, hydrogen-poor object bright in the far UV. The observed light changes and spectrum changes are produced as the small star passes in front of the large star near phase $0^{P} 0$. Around phase $0^{P} .5$ the small star is eclipsed, the eclipse having long partial phases. The He I lines then weaken and the sharp lines strengthen, the latter being formed as the atmospheric eclipse proceeds. We have measured a series of spectrograms obtained in 1972, May and June for radial velocity. A preliminary look at these results supports the interpretation given here. Neither star is a normal object. Possibly the small star has just finished ejecting its hydrogen rich envelope onto its companion.

Thackeray: We have just a little material on this event, which we examined after the discovery of the helium variation. We definitely found evidence that the $\mathrm{N}$ II lines appeared to be varying in opposite phase. This agrees physically with your remarks about C II: you seemed to be a little doubtful about that group (iii).

Cowley: What can you say about the mass ratio and the mass function?

Underhill: Nothing! The radial velocities measured on any one night show a wide scatter. A trend over the 8.8 period seems to be present. The range isn't more than $15 \mathrm{~km} \mathrm{~s}^{-1}$ at the most. The narrow lines and the hydrogen lines vary by something like $5 \mathrm{~km} \mathrm{~s}^{-1}$.

Cowley: Are your plates of high enough dispersion to determine whether the spectrum shows ${ }^{4} \mathrm{He}$ or ${ }^{3} \mathrm{He}$ ?

Underhill: If there were ${ }^{3} \mathrm{He}, \lambda 6678$ would have a sizeable displacement to the red. I have measured all the spectrograms of the red and yellow regions, which have a disperison of about $12 \AA \mathrm{mm}^{-1}$. The He I line at $\lambda 6678$ seems to have the same sort of variation as the rest of the $\mathrm{He}$ I lines have. I would say there is no ${ }^{3} \mathrm{He}$.

Hutchings: Is there any photometry on this star?

Underhill: The only photometry I know that has been published is that of Norris - which is very little. Rudolph Schild told me he had been observing the star in $U, B, V$, and that he found little, if any, variation.

Wright: Is $\lambda 4383$ the only neutral iron line that you have observed? Your plate looks as if it is fairly strong.

Underhill: No, it's not the only one. It's the easiest to put in a diagram. When $\lambda 4383$ is strong, all the major Fe I lines are present.

Wright: So you can measure quite a number of them.

McNamara: Any additional questions? Thank you for a very interesting talk. Now Dr. Andersen will speak about SX Cas.

Andersen: The eclipsing binary SX Cas was first extensively observed by Struve (1944). The period is $36^{\mathrm{d}} 6$, the spectral types approximately A6 and G6 with giant characteristics. Struve's velocity curve showed abnormally large scatter and $e \cos \omega$ 
close to 0.5 , in violent disagreement with the photometric result that $e \cos \omega=0$. From this, Struve inferred the now well-known type of distortion of the velocity-curve by absorption from a gas stream, which also gives rise to strong emission at $\mathrm{H} \alpha$ and $\mathrm{H} \beta$. During the entire cycle, Struve noted the weakness of the Si II lines and $\mathrm{Mg}$ II $\lambda 4481$ and interpreted this as dilution effect and the spectrum as arising in a shell. Dr. Batten's collection of plates of SX Cas have been studied during a visit to the Dominion Astrophysical Observatory, both as regards selection and measurement of a number of reliable lines in order to obtain a good radial-velocity curve for the system and to detect possible differences between various ions, as well as to measure more suspect lines ( $\mathrm{H}, \mathrm{Si}$ II, Mg II). The profiles of the Balmer emission lines have also been obtained. A few points of interest have emerged:

(1) Strong changes in the emission profiles have been noted at the same phase in cycles separated by a year or two, and smaller but significant changes occur from one night to the next at a phase at which the geometry of the system and the velocities change the least, and where the total light is constant. Struve's observations extended over three cycles only, to minimize the effect of these changes.

(2) The secondary spectrum has been detected, and the few available points on the velocity curve indicate amplitudes of about $K_{1} \simeq 45 \mathrm{~km} \mathrm{~s}^{-1}$ and $K_{2} \simeq 90 \mathrm{~km} \mathrm{~s}^{-1}$. These values are rather uncertain, especially that for $K_{1}$, as the velocities of the primary show a much larger scatter than the observational uncertainty justifies. The velocity curve leads to two inferences:

(a) with a mass ratio $m_{2} / m_{1} \simeq 0.3-0.5$, the range indicated by the velocities, the radii of the Roche lobe are as given in Table $I$. Comparison with the fractional radi

TABLE I

Possible Parameters for SX Cas

\begin{tabular}{llll}
$m_{1} / m_{2}$ & 0.3 & 0.5 & $r_{\mathrm{ph}}$ \\
& & & \\
$r_{\mathrm{p}}$ & 0.49 & 0.44 & 0.08 \\
$r_{\mathrm{s}}$ & 0.27 & 0.31 & 0.21 \\
\hline
\end{tabular}

obtained from the light curve shows the primary well within its lobe, leaving space for the gas ring proposed by Günther (1959). The present data seem to indicate that the secondary does not fill its Roche lobe, despite the substantial amount of circumstellar matter in the system, but the scatter in the velocities, especially of the primary, makes this conclusion uncertain.

(b) The total mass of the system appears to be around $8 M_{\odot}$, with $5 M_{\odot}$ to $6 M_{\odot}$ for the primary, which is too high for a main-sequence late A-type star. However, the observed spectrum of the primary is clearly not a normal stellar spectrum, but a shell spectrum with weak Si II and $\mathrm{Mg}$ II $\lambda 4481$, and the Balmer lines frequently visible to about $\mathrm{H30}$, the higher Balmer lines being extremely sharp. The spectrum is strongly variable in character, sometimes having very sharp and deep absorption lines, at 
other times showing only wide, shallow lines of complicated structure. The changes can be seen on plates with only little separation in phase, but cycle-to-cycle variations may play a role. The primary thus has an extended envelope dominating its spectrum, apparently to the extent of concealing the spectrum of the primary star itself.

Underhill: Have you observed the primary spectrum in the red?

Andersen: Apart from the emission at $\mathrm{H} \alpha$, no. The plates we have were obtained mainly in order to study this emission. Even at $30 \AA \mathrm{mm}^{-1}$, a IIa-F plate needs about four hours exposure. The continuum is very weak, especially near the $D$ lines, but there are some measurable iron lines from the secondary spectrum around $\lambda 6400$.

Hall: You mentioned that the cool star apparently does not fill its Roche lobe. Whenever the radial velocity curve of the hotter star is distorted in such a way as to give a spurious eccentricity, Struve thought that in general the value of $K_{1}$ was exaggerated. Hardie (1950) tried to remove the distortion in the velocity curve of $U$ Cep, found a circular orbit, and in the process he reduced $K_{1}$. I think that was the only quantitative investigation of this problem. If $K_{1}$ could be decreased for SX Cas, you would get a mass ratio farther away from one, and this would make the fractional radius of the Roche lobe smaller.

Andersen: As I understand it, Hardie tried to separate the spectrum of the stream from that of the star. We got high-dispersion plates of SX Cas for the same purpose. Now our two plates of highest dispersion are at 0.83 and $0^{P} .9$ - just the phases where Struve found the maximum distortion. Now the plate of 0.83 does not show any asymmetric profiles - all the lines are wide. There is a suggestion of asymmetry on the plate at 0.9 , but I measured only the wide lines - both plates were measured on an oscilloscope machine. It so happens that we have as yet few plates covering the distorted part of the velocity curve, and the only advantage of this is that our value of $K_{1}$ should be relatively unaffected by the distortion.

Batten: As far as U Cep is concerned, I think the value of $K_{1}$ should be increased again. I believe that the particular way in which Hardie attempted to separate the spectra of stream and star was based on a wrong assumption - at least for U Cep. I'm not at all sure that it is a safe generalization to say that removing the distortion by the stream will reduce the value of $K_{1}$.

McNamara: In the spectrum of U Sge the lines other than hydrogen give quite different velocities from those given by the hydrogen lines. Is this also true for U Cep?

Batten: There are few lines other than those of hydrogen, in the spectrum of $U$ Cep, that are measurable at the dispersion I have used.

Cowley: I think the fact that Dr. Andersen finds the same $\gamma$-velocity for both components and the zero eccentricity suggests that most of the distortion of the velocity curve has been removed.

Andersen: I have measured 75 lines on the plates, in order to obtain decent mean velocities for each ion. There appears to be little or no difference between ions. At some phases we see emission in lines other than those of hydrogen.

Hall: This is a bad problem and unfortunately I don't have the final answer yet. But there is circumstantial evidence to suggest that the source of the problem lies 
somehow in the radial velocity curve of the primary star. Look at the list of Algol-type binaries in which this problem exists, i.e., in which the subgiant appears not to fill its Roche lobe. Exclude from this list the binaries of the RS CVn type, which are fundamentally different. Excluding these is important because in these binaries the subgiants very definitely, and by a large margin, do not fill their lobes. Then if you look at the remaining candidates, you find that the binaries giving you the most trouble are those in which the overall amplitude of the radial velocity variation of the primary star is smallest relative to its scatter; at least Struve's radial-velocity curves show this, and they are still the only ones available. An example is RW Per in which the subgiant seems to be smaller than its Roche lobe by quite a significant margin, but in this case there is almost no amplitude to the radial velocity curve of the primary - the variation is almost all scatter.

Thus it is my feeling that the Algol-type binaries with 'undersize' subgiants can be explained as follows: those in which the subgiants clearly do not fill their Roche lobes are not really Algol-type (because they are RS CVn-type instead) and those which clearly are Algol-type have distorted radial-velocity curves and hence erroneous mass functions which make it appear that the subgiants are smaller than their Roche lobes whereas in fact they fill their lobes.

Andersen: Although the scatter is very large, the internal mean error of the individual plate velocities is only about $2 \mathrm{~km} \mathrm{~s}^{-1}$. I have tried to estimate the minimum mass ratio, that I thought I could get from the velocity curve, and even with that, the Roche lobe is at least $33 \%$ larger than the secondary component.

Thackeray: I believe that Struve's curve is based on a massive series of observations in one cycle.

Andersen: Three consecutive cycles in the summer of 1943.

Thackeray: I suppose it is possible that gaseous-stream activity was unusually strong then.

Sahade: From your description of the spectrum you seem to have a shell spectrum, and you have somehow to make sure that you are measuring the stellar line.

Andersen: I don't really believe that I am at any point measuring stellar lines - but from the kind of variation I think I am measuring the spectrum of something that moves with the primary star.

Sahade: Shell lines in a binary spectrum may move in phase with one of the components, and yet have an amplitude different from that of the orbital motion of the star.

Andersen: How can we explain the spectrum of the primary star? Can we have a massive star surrounded by a shell that makes it look like an A-type star? The shell is dense enough to hide the primary completely, but still dilute enough to show the high Balmer lines.

Plavec: Didn't Struve classify the stars as giants? He added the prefix c to the spectral types, indicating high luminosity. If the primary were a bright giant, then it could have five or six solar masses at its place in the $\mathrm{H}-\mathrm{R}$ diagram.

Andersen: The prefix c indicated very narrow lines - but these are the shell lines. We don't know how luminous the star is. 
Plavec: The problem is similar to that of V367 Cyg.

McNamara: Although we have deliberately let the discussion go on, three more people want to speak this afternoon. We have a series of contributions on VV Cep and the $\zeta$ Aur stars.

Wright: I should like to present a few preliminary results on measures of the $\mathrm{H} \alpha$ line in the spectrum of VV Cep as observed for the past fifteen years. An orbit for the system and analysis of the emission profile as observed near eclipse has been given by Hutchings and Wright (1971). In order to derive the emission wings in the spectrum of the early-type star, it was necessary to subtract the profile of the spectrum of $\alpha$ Ori from that of VV Cep over about twenty Ångstroms, but the results are remarkably consistent. In order to disentangle this complex spectrum further, the additional lines at $\mathrm{H} \alpha$ were plotted with the $\mathrm{H} \alpha$ emission line as a continuum and velocities were measured relative to the undisplaced centre of the $\mathrm{H} \alpha$ line after orbital motion was removed. The principal, fairly definite feature, is a sharp absorption line that is observed over the whole cycle with velocity close to the velocity of the system, about $-20 \mathrm{~km} \mathrm{~s}^{-1}$. After secondary eclipse, near J.D. 2438 700, a fairly sharp emission feature can be seen on nearly every plate, with velocities about $-60 \mathrm{~km} \mathrm{~s}^{-1}$. Similar emission lines can be seen in the ultra-violet region of the spectrum. Fairly strong absorption lines have also been measured on all plates. The velocities are about $0 \mathrm{~km} \mathrm{~s}^{-1}$ from primary to secondary eclipse and increase to about $30 \mathrm{~km} \mathrm{~s}^{-1}$ from secondary eclipse to the present. These lines become double near mid-eclipse and are probably related to mass motions between the stars and around the secondary star, though a detailed model is not yet available. Numerous other weak lines, with velocities up to $\pm 250 \mathrm{~km} \mathrm{~s}^{-1}$ also have been measured.

Faraggiana: Several spectra have been taken since 1967 at the coudé foci of the $193 \mathrm{~cm}$ and $152 \mathrm{~cm}$ telescopes of the Haute Provence Observatory with dispersions of $9.7 \AA \mathrm{mm}^{-1}$ and $12.4 \AA \mathrm{mm}^{-1}$, covering the region $\lambda \lambda 3500-7000$. The radial velocities have been measured with an impersonal device that gives the positions of the lines with an error of one or two microns. The radial-velocity curve of the M-type star has been derived, both in the red and blue regions of the spectrum, from the lines of the neutral metals $\mathrm{Cr}, \mathrm{V}, \mathrm{Fe}$ and $\mathrm{Ni}$. The results have been plotted with the previous ones of Wright and Larson (1969) on the curve computed by Hutchings and Wright (1971). The agreement between ours and the Victoria measures appears very good. In Figure 1 we have compared our measurements with those of Peery (1966). All his radial velocities are less negative than ours at the corresponding phases. It is possible that the difference $\Delta v$ at the minimum of radial velocity (with $5 \mathrm{~km} \mathrm{~s}^{-1}<\Delta v<10 \mathrm{~km}$ $\mathrm{s}^{-1}$ ) is real. The only ionized element belonging to the M-type star is Ba I as indicated by the radial velocity of the three lines $\lambda \lambda 6496,4934$, and 4554 .

Emission lines of FeII and [Fe II] are present and according to McLaughlin (1951) show radial velocities close to that of the centre of mass of the system indicating that they are formed in an envelope surrounding the system. Our radial velocities from lines of $\left[\mathrm{Fe}\right.$ II] are effectively nearly constant at $-20 \mathrm{~km} \mathrm{~s}^{-1}$; the radial velocities from lines of $\mathrm{Fe}$ II are variable. We have measured the sharp core of the emission which 
208

SEVENTH DISCUSSION SESSION

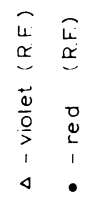

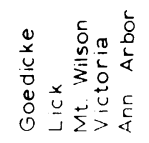

$$
x+0 \cdot 0
$$

$\square$

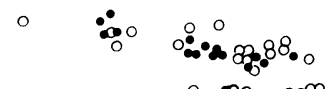

$\triangleleft$

๑.

$\triangleleft \triangleleft$

$\checkmark$

$$
\text { - } .0 .000
$$

no 800

- 0 \%880 ०。

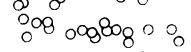

$80.8800000 \%$

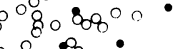

$\infty$

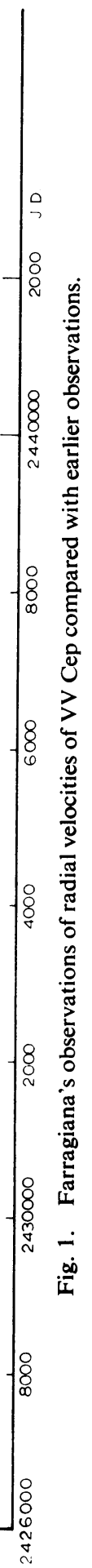

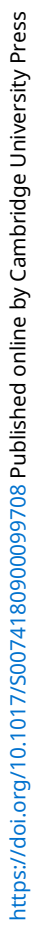




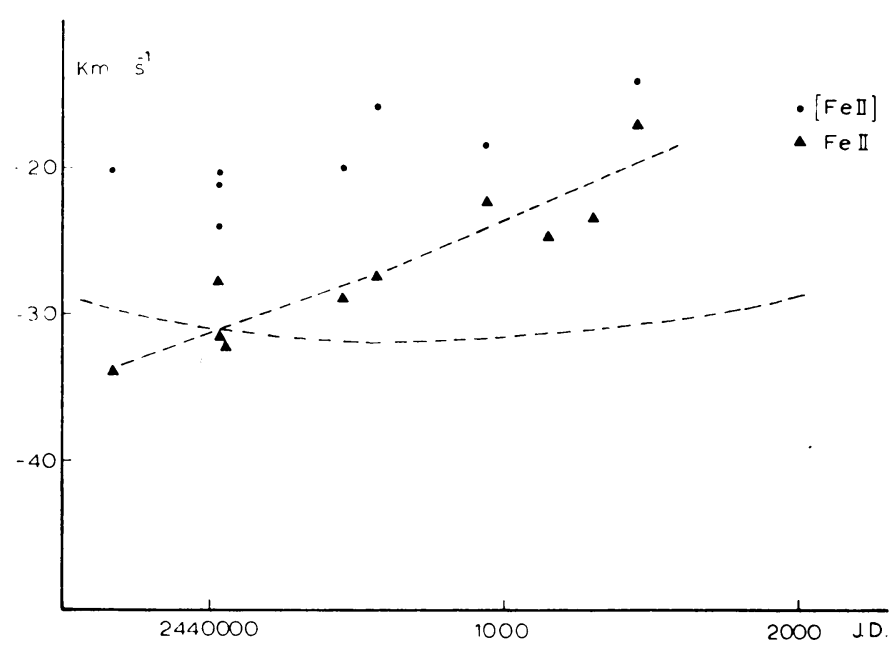

Fig. 2. Radial velocities derived from lines of $\mathrm{Fe} I I$ and $[\mathrm{Fe} \mathrm{II}]$ in the spectrum of VV Cep, compared with the stellar velocities.

is well visible and measurable on all our spectrograms. The Fe II curve cuts the curve derived from the M-type spectrum at J.D. 2440000, and becomes increasingly more positive with respect to that curve (Figure 2). Hence it does not seem to indicate the motion of the B-type companion because in this case the maximum radial-velocity should have been expected around J.D. 2440000 . From a preliminary visual inspection the intensity ratio $\lambda 4233 \mathrm{Fe} \mathrm{II} / \lambda 4243[\mathrm{Fe} \mathrm{II}]$ is variable reaching a maximum value in 1969-1970. The absorption cores of the first Balmer lines show a radial-velocity progression, $\mathrm{H} \alpha$ giving a more negative velocity. The values of radial-velocity of the higher members of the series ( $\mathrm{H} 9$ to $\mathrm{H} 17)$ are almost constant and range from slightly more than zero (1967-1968) to about $+20 \mathrm{~km} \mathrm{~s}^{-1}$ in 1972. The amplitude of the radial-velocity progression $\mathrm{H} \alpha-\mathrm{Hn}$ increases from about $-30 \mathrm{~km} \mathrm{~s}^{-1}$ in $1967-1968$ to about $-40 \mathrm{~km} \mathrm{~s}^{-1}$ in 1970-1972. Two spectrograms show a different behaviour: GB 1246 (1971, July 25) and GB 1736 (1971, December 17): a sharp discontinuity of about $40 \mathrm{~km} \mathrm{~s}^{-1}$ between $\mathrm{H} \gamma$ and $\mathrm{H} \delta$ is observed and the radial-velocities of the higher members of the series are slowly decreasing toward the mean value given by the other spectrograms.

The radial-velocity of the absorption core of $\mathrm{H} \alpha$ has been plotted on the graph published by Wright and Larson. The zero velocity is that of the M-type star. Our data are very well connected with the previous Victoria observations. The emission radial-velocity of $\mathrm{H} \alpha$ has been measured by taking the centre of the interpolated emission contour and by measuring the shift with respect to the absorption core. The results are plotted on the previous graph: also in this case a good connection with the Victoria data is found.

Wright: A coordinated program for observing eclipses of the $\zeta$ Aur stars, sponsored by IAU Commission 42, has just been concluded. I should like to summarize 
the observations and then additional comments will be made by Dr. Wood and Dr. Kitamura. The program began with 32 Cyg in 1971, October-November. The most extensive observations were obtained in Japan at Dodaira, Okayama on Akita. The Japanese observers showed that, this time at least, the eclipse was not total and, indeed, Saito and Sato showed that at mid-eclipse about half the disk of the B-type component is eclipsed by the K-type star. Using data from previous eclipses, a period of $1147.20 \pm 0.25$ has been derived. Spectroscopic observations were obtained at Okayama, Haute Provence and Victoria, and at least seven other groups, obtained photometric observations.

The eclipse of $\zeta$ Aur was also well-observed, both photometrically and spectroscopically by the Japanese observers. The most interesting phases were from 1971, November 26 to 1972, January 7. A good series of spectra during egress was obtained by Simon in Hawaii.

In the spring, 31 Cyg was in eclipse. Complete results have not yet been received but the reported observations show that the period of $3784^{\mathrm{d}}$, derived from the 1951 and 1961 eclipses, is confirmed. Good series of spectra were obtained in Japan, especially during ingress and at Victoria, especially during egress.

I should like to extend special thanks on behalf of Commission 42 to all observers in this successful program.

Wood: I want to speak primarily about observations of $31 \mathrm{Cyg}$ and $32 \mathrm{Cyg}$. While I'm on my feet, however, I'd like to mention the preprints of a paper by D. Schuerman

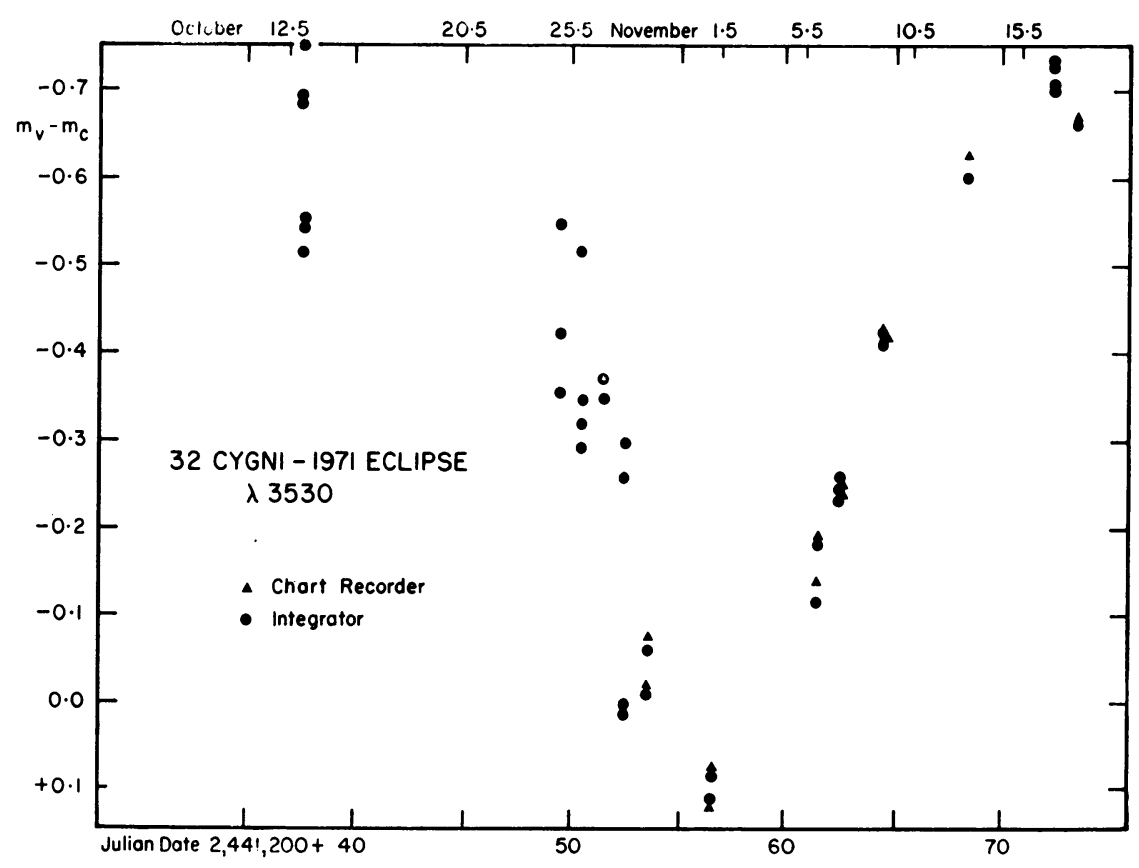

Fig. 3. Florida observations of the 1971 eclipse of 32 Cyg. 
TABLE II

Eclipses of 31 Cyg and 32 Cyg

\begin{tabular}{|c|c|c|}
\hline \multirow{2}{*}{$\begin{array}{l}\text { Wavelength } \\
\AA\end{array}$} & \multicolumn{2}{|c|}{ Depth of Eclipse } \\
\hline & $\begin{array}{l}31 \text { Cyg } \\
m\end{array}$ & $\begin{array}{l}32 \text { Cyg } \\
m\end{array}$ \\
\hline 3530 & 1.83 & 0.76 \\
\hline 4240 & 0.67 & 0.28 \\
\hline 5000 & 0.16 & 0.11 \\
\hline
\end{tabular}

For 31 Cyg, egress from totality began between July 1.32 and 2.31 (UT). Eclipse ended in yellow by July 6.14 , but still down by $\sim 0$ m08. Totality began between April 28.4 and May 2.4 (all dates 1972).

that several of us have received here. He considers the effect of radiation pressure on the Roche potentials and finds that some systems in which one component is very luminous may have no contact surface. This is perhaps relevant to some of our discussions. At Rosemary Hill Observatory R. H. Bloomer and others have observed 32 Cyg with three narrow-band filters prepared by Gyldenkerne for the collaborative campaign. The observations are shown in Figure 3 and Table II. These observations seem, at first sight, to agree well with Japanese observations reported by Dr. Wright. All observations together should establish well the parameters during eclipse. Apparent intrinsic fluctuations during eclipse are probably observational scatter. The duration of eclipse and the period were so poorly known that observations were made on nights of dubious photometric quality since even a crude indication of the phase of the eclipse would be better than nothing. We have already reported to Dr. Wright on possible rapid variations (in an hour or two) in the ultra-violet ( $\lambda 3536)$ region. We have nothing to add and are greatly interested in learning of other observations.

Bloomer, D. H. Martin, and myself have been observing $31 \mathrm{Cyg}$. Our results are summarized in Table II. The observations immediately preceding eclipse are of particular interest. In Filter No. 6 ( $\lambda 5000$ narrow-band) on four nights in April the observations agree well. On April 26 UT (JD 2441433), the first two observations averaged 0.02 brighter, but four others agreed with the earlier ones. On April 28 UT (JD 2441435) the first four observations average 0.02 fainter as though eclipse had begun but the last four (looking quite good) made about $45 \mathrm{~min}$. later showed the system more than 0.1 brighter.

In Filter No. 5 ( $\lambda 4240$ narrow-band) again the first four nights in April were extremely congruent with the variable $1^{\mathrm{m}} .15$ brighter. On April 26 , the average of seven observations were slightly more than 0 m 06 fainter, suggesting that eclipse was beginning in this wavelength. On April 28, the observation began with $\mathrm{C}-\mathrm{V}=1^{\mathrm{m}}$.05 (variable brighter), dropped in $40 \mathrm{~min}$. to 0.90 and this increased in the next $30 \mathrm{~min}$. to $1{ }^{\mathrm{m}} \cdot 13$. 
Observations in Filter No. 4 ( $\lambda 3530$ narrow-band) gave quite constant results on four nights from April 13-25. On April 26, there was a steady increase from a $\Delta m$ of $1^{\mathrm{m}} .60$ at $07^{\mathrm{h}} 00^{\mathrm{m}}$ UT to $1^{\mathrm{m}} .81$ at $09^{\mathrm{h}} 20^{\mathrm{m}}$; then on April 28 , in a pattern similar to the other colours, the measured magnitude difference ranged from $1^{\mathrm{m}} .75$ at about $06^{\mathrm{h}} 30^{\mathrm{m}}$ UT ( 3 observations) to $1^{\mathrm{m}} \cdot 67$ about $07^{\mathrm{h}} 20^{\mathrm{m}}$ ( 5 observations) to $1^{\mathrm{m}} .82$ at $09^{\mathrm{h}} 00^{\mathrm{m}}(6$ observations). There may have been an eclipse in the red. The readings in April were rather constant at $\mathrm{C}-\mathrm{V}=1^{\mathrm{m}} .49$; on May 5 they were $1 . \mathrm{m} \cdot 46$ and on May 22 between $1 \mathrm{~m} .44$ and $1 . \mathrm{m} .45$. If this is an eclipse effect, the depth is $0^{\mathrm{m}} .04$. The observations during totality were extremely constant on any given night in all colours. The observations in the $3530 \AA$ region, however, suggest a slow brightening during eclipse of about 0.15 . We will discuss the evidence in more detail when the complete description of the observations is published.

Hall: I have some photometric observations of $31 \mathrm{Cyg}$ and $\zeta$ Aur. They were obtained in $U, B, V$ bands by Larry P. Lovell, an amateur astronomer. I know he got at least one observation on the downward branch of the light curve, three on the upward branch, and several in totality. People might like to know that these observations were made.

Kitamura: Between September of 1971 and January of 1972, more than three hundred differential $U B V$ photoelectric observations of $\zeta$ Aur were made in Japan. The photoelectric observations were carried out with the $91-\mathrm{cm}$ reflector at the Dodaira Station of Tokyo Astronomical Observatory, the $30-\mathrm{cm}$ reflector at the Okayama Astrophysical Station of the same Observatory, the $25-\mathrm{cm}$ reflector at the

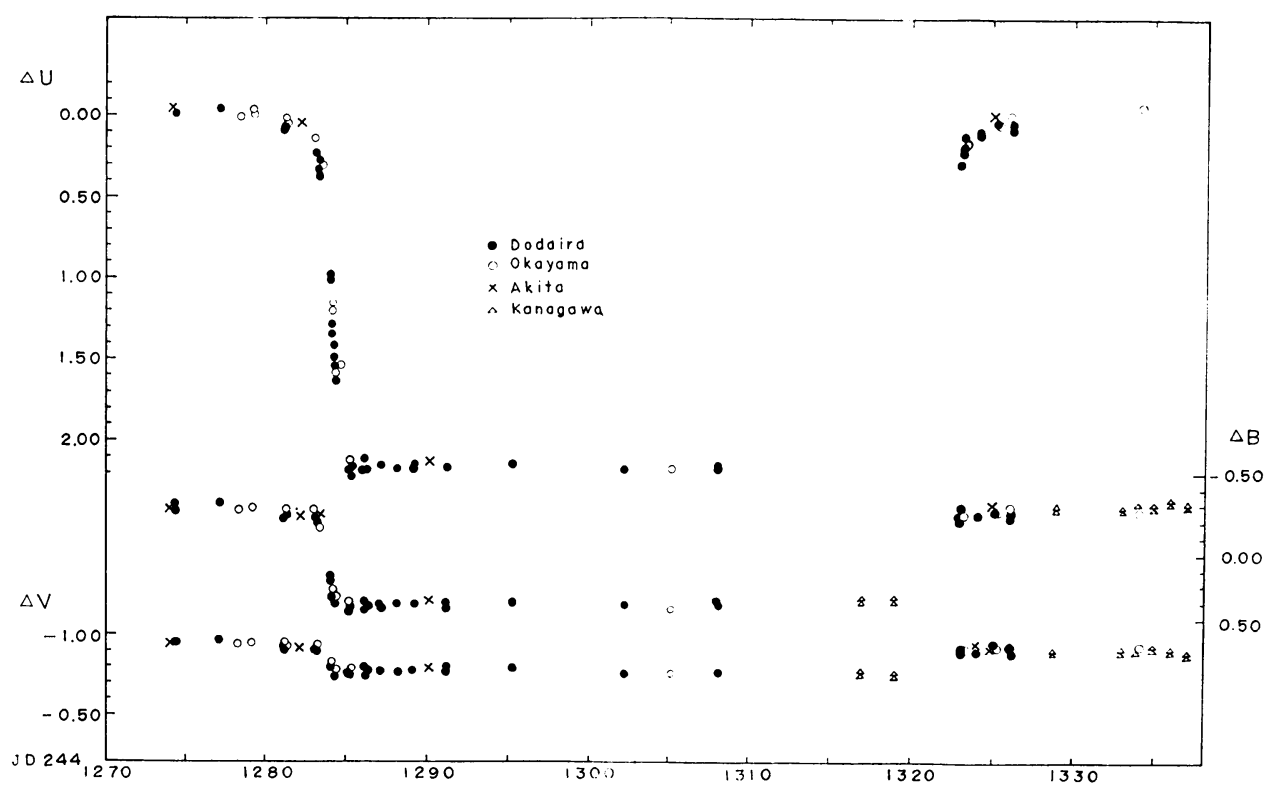

Fig. 4. Light variations of $\zeta$ Aur compared with $\lambda$ Aur between JD 2441274 (1971, Nov. 18) and JD 2441337 (1972, Jan. 20) observed from Japan. 
Akita University and the 20-cm refractor at the Education Centre of Kanagawa Prefecture. These cooperative observations were undertaken with the purpose of covering as many phases as possible during the eclipse. Observations were made on forty-eight different nights, differentially with respect to $\lambda$ Aur. Figure 4 shows light variations of $\zeta$ Aur $-\lambda$ Aur, in the Johnson system. General features of the light curve are similar to those in previous eclipses, but in the present eclipse the depths are s.ystematically greater by $0^{\mathrm{m}} .04$ to 0.06 irrespective of the $U B V$ colours.

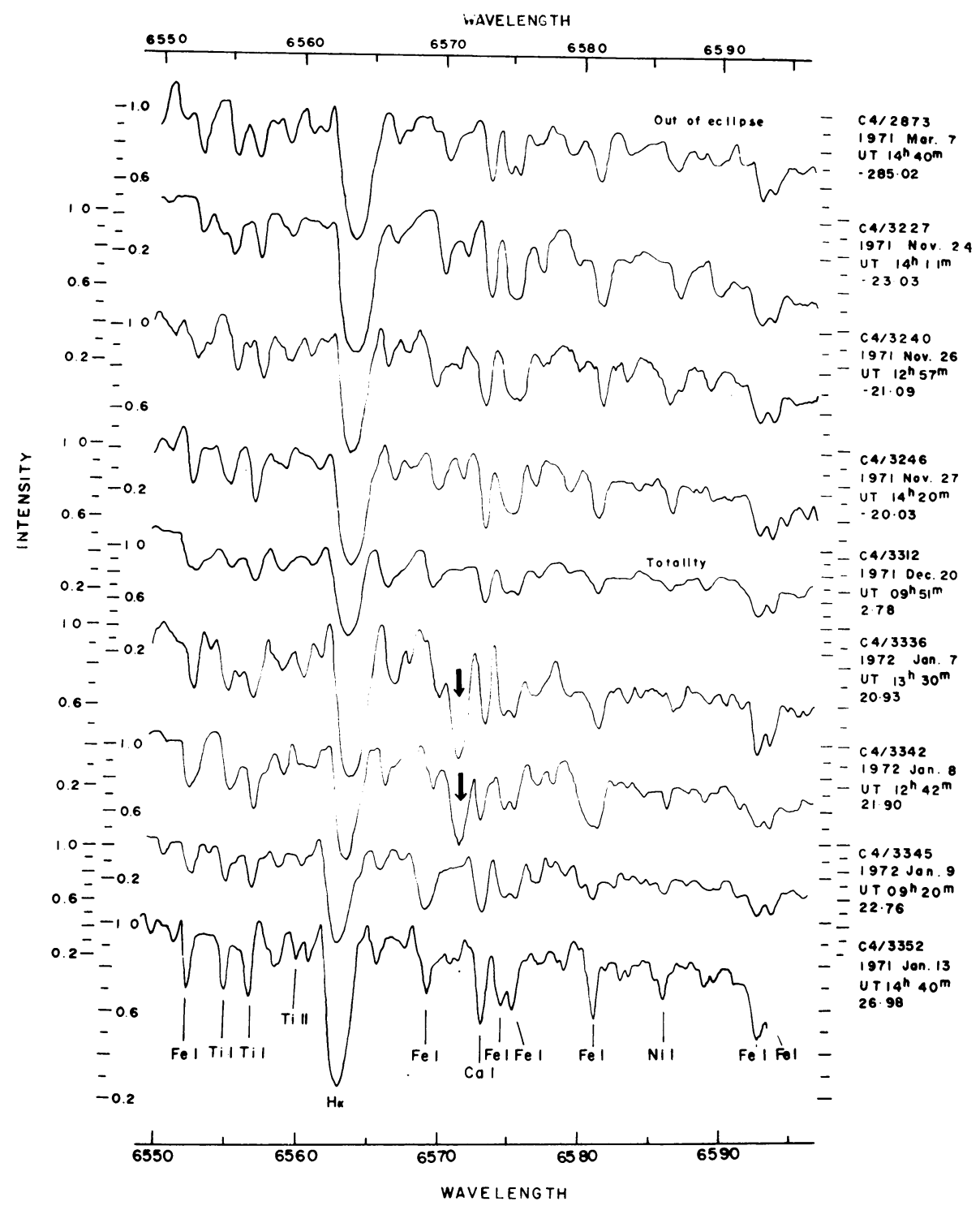

Fig. 5. Intensity tracings of spectrograms of $\zeta$ Aur obtained by M. Saito in the fall and winter of 1971-1972. The arrows point to the satellite lines of Ca I at $\lambda 6572$. 
Simultaneously, spectroscopic observations were made with the 199-cm (74-inch) reflector at Okayama mainly by $M$. Saito, and 60 coudé spectrograms were obtained. Of these spectrograms, 20 were taken at the $\mathrm{H} \alpha$ region with a dispersion of $20 \AA \mathrm{mm}^{-1}$ and 40 in the blue and ultra-violet regions with the dispersion of $10 \AA \mathrm{mm}^{-1}$. Figure 5 shows some interesting phenomenon that appeared at $\mathrm{Ca}$ I ( $\lambda 6572)$, on the spectrograms taken around the fourth contact. The same thing can be seen at $\mathrm{Ca}$ I $(\lambda 4227)$ on the spectrograms taken on the same nights. These would indicate strong satellite

TABLE III

Radial velocities of satellite lines of $\mathrm{Ca}$ I

\begin{tabular}{|c|c|c|c|c|}
\hline \multirow[t]{2}{*}{ Pl. No. } & \multirow[t]{2}{*}{ Date } & \multirow[t]{2}{*}{ Phase } & \multicolumn{2}{|l|}{ Radial velocities } \\
\hline & & & Main Line $\lambda 6572^{a}$ ) & Main line $\left.\lambda 4226^{b}\right)$ \\
\hline $\mathrm{C} 4 / 3336$ & 1072, Jan. 7 & $29 d 93$ & $-90 \mathrm{~km} \mathrm{~s}^{-1}$ & - \\
\hline C4/3337 & 1972, Jan. 7 & $21 \stackrel{d}{0} 00$ & - & $-57 \mathrm{~km} \mathrm{~s}^{-1}$ \\
\hline $\mathrm{C} 4 / 3340$ & 1972, Jan. 8 & $21 \mathrm{~d} 77$ & - & $\left.-23 \mathrm{~km} \mathrm{~s}^{-1 \mathrm{c}}\right)$ \\
\hline $\mathrm{C} 4 / 3341$ & 1972, Jan. 8 & $21+90$ & $-74 \mathrm{~km} \mathrm{~s}^{-1}$ & - \\
\hline
\end{tabular}

a Velocity displacement from the $\mathrm{K}$ star's lines

b Velocity displacement from the chromospheric lines

c Faint

lines. The velocities are given in Table III. The detailed reduction of all the spectrograms is now in progress.

Wright: I'd like to make a comment about this chromospheric line of $\mathrm{Ca} \mathrm{I}$ in the spectrum of $\zeta$ Aur. It's the intercombination line, and therefore it could be produced in relatively low-density populations. I'm quite surprised that it is so prominent on these particular plates, because at $\mathrm{H} \alpha(\lambda 6500)$ the B-type star is very, very difficult to see at any time, and especially outside eclipse. This certainly seems to be a chromospheric effect. As far as I am aware, nothing like it has been detected before in this particular region of the spectrum.

Whelan: I would just like to underline Professor Wood's mention of Schuerman's paper. Taking into account the effects of radiation pressure on particles may be an important theoretical advance.

McNamara: If there are no more comments or questions this afternoon, I'd like to thank those who have participated, and close this session.

\section{References}

Binnendijk, L.: 1963, Astron. J. 68, 22.

Binnendijk, L.: 1965, Kleine Veröffentl. Remeis-Sternw. 4, 36.

Broglia, P.: 1960, Contr. Oss. Milano-Merate No. 165.

Grygar, J.: 1972, Bull. Astron. Inst. Czech. 23, 175.

Günther, O.: 1959, Astron. Nachr. 285, 97, 105.

Hall, D. S. and Garrison, L. M.: 1972, Publ. Astron. Soc. Pacific 84, 552 
Hardie, R. H.: 1950, Astrophys. J. 112, 542.

Hazlehurst, J.: 1970, Monthly Notices Roy. Astron. Soc. 149, 129.

Hutchings, J. B. and Hill, G.: 1973, Astrophys. J. 179 (in press).

Hutchings, J. B. and Wright, K. O.: 1971, Monthly Notices Roy. Astron. Soc. 155, 203.

Klinglesmith, D. A., Bernacca, P. L., and Frey, H.: 1971, Veröffentl. Remeis-Sternw. 9, 205.

Lucy, L. B.: 1968, Astrophys. J. 153, 877.

McLaughlin, D. B.: 1951, Astrophys. J. 114, 47.

McNamara, D. H.: 1967, Astrophys. J. 149, 723.

Mauder, H.: 1972, Astron. Astrophys. 17, 1.

Miner, E. D.: 1966, Astrophys. J. 144, 1101.

Molnar, M.: 1972, private communication to A. B. Underhill.

Moss, D. L. and Whelan, J. A. J.: 1970, Monthly Notices Roy. Astron. Soc. 149, 147.

Mochnacki, S. W. and Doughty, N. A.: 1972, Monthly Notices Roy. Astron. Soc. 156, 51, 243.

Norris, J.: 1971, Astrophys. J. Suppl. 23, 235.

Peery, B. J.: 1966, Astrophys. J. 144, 672.

Ruciński, S. M.: 1969, Acta Astron. 19, 125, 245.

Struve, O.: 1944, Astrophys. J. 99, 89.

Wright, K. O. and Larson, S. J.: 1969, in M. Hack (ed.), Mass Loss from Stars, Reidel-Dordrecht, p. 198. 